\title{
Gamification for Assessment of Customer Care Employees
}

\author{
A.V. Murali \\ Software Consultant
}

\begin{abstract}
Gamification is a buzzword today in most organizational settings. On the other hand, on-the-job assessments are carried out in several organizations on a regular basis. Assessments are meant to ensure that employees are up-to-date in their knowledge of the work domain. Traditionally assessments are carried out using an online platform where an employee would login to the system and answer a set of questions posed by the system. In this paper we propose alternate methods of assessment based on gamification. Ten different approaches have been suggested for gamified assessment. The ideas are particularly suited to customer care employees who are under constant pressure to meet their performance goals.
\end{abstract}

Keywords: Gamification, Assessment, Customer Care, Gamification Modes, Game Mechanics

\section{Introduction}

Customer service representatives are under constant pressure to provide outstanding customer experience. They are expected to have certain traits, characteristics and behaviors that go towards making the customer feel special, valued and respected. They are expected to possess correct, complete and up-to-date knowledge of the products, the processes and the services of the organization they work for. In addition, they are expected to maintain certain level of performance, in terms of number of calls they attend per day, their average handling time and the number of first call resolution. Working in shift adds further stress to their physical health. They undergo soft skills training so that they would be better equipped to handle irate customers.

Customer service representatives undergo assessments at various stages from the moment they join an organization. They are regularly assessed on various metrics to ensure that they remain up-to-date and provide superior service to the customers.

\section{Literature Survey}

\subsection{Traditional assessment methods}

In a traditional assessment paradigm, an examinee would login to an assessment tool from their desktops. The assessment tool would pick questions from its pool of question and pose them to the examinee in some sequence. Two types of assessments are popular in many work setups. One is static in nature in the sense that the assessment engine starts from the question that is marked as first in its database, gets examinee's response, moves on to second and so on till all the questions are exhausted. Another one is adaptive in nature [1] in the sense that it adapts its question sequence in tune with the examinee's knowledge level. It typically starts with a question of medium level difficulty. If the student could answer it then it poses a relatively difficult question. But if the student could not answer it then it moves to a relatively easier question. This way its selection of a question at any point of time during the assessment session is dynamic in nature and it depends on the examinee's performance so far. There are merits and demerits to both the approaches and the selection of the method depends on the domain of assessment and the nature of questions.

\subsection{Gamification}

Gamification is the application of game-design principles to change non game-like situations [2] into fun and engaging game-like environments for the purpose of motivating and changing employee attitude and behavior. Gamification has been applied to several work settings across industries with positive results.

Game mechanics are constructs of rules or methods designed for interaction with the game state, thus providing gameplay [3]. Game mechanics are mechanisms utilized by game designers to reward activity among people for carrying out their daily routine. Some of the common game mechanics widely employed in industries are points, achievement badges, levels, rewards, leaderboard, quests, challenges, feedback loops and progress bar [4]. The target group could be employees of an organization, its customers and other classes of users. Game mechanics are intended to bring about a highly motivating experience for the target group of people. Many surveys have been conducted to find out the effectiveness of gamification on the work environment. A large percentage of respondents were of the view that everyday activities turn more fun and rewarding when they are made more like a game. People tend to keep a closer watch on their activities if a layer of competition is added to their everyday routine.

There are several benefits of gamification. It encourages joyful optimism, fun, intense focus and competitiveness. Further, it has been found to improve productivity, retention and collaboration. It encourages friendly competitions with peers.

\section{Problem Definition}

As noted earlier, customer service representatives undergo enormous amount of work pressure in their daily routine. In 


\section{International Journal of Science and Research (IJSR) \\ ISSN (Online): 2319-7064}

Index Copernicus Value (2013): 6.14 | Impact Factor (2015): 6.391

such a high-pressure scenario, regular assessments can only add to the already existing work burden. The paper seeks to find ways of carrying out assessments that can mitigate the added pressure that generally goes with assessments.

Regular assessments can be demotivating, boring and an anxiety-ridden experience in a scenario like this where performance is monitored and measured from various perspectives. The purpose of this work is to come out with methods that can add fun element to the assessment sessions. Assessments should not only be fun but rewarding too. With this in mind, the author looks at gamification as a tool to achieve the objective of adding fun element to assessments. The author believes that adding fun to assessment not only encourages customer care representatives to participate willingly in such assessments but also feel motivated to work and perform better.

\section{Methodology and Approach}

As gamification has been found to have several merits, the author has explored the possibility of applying gamification techniques to assessment of employees. While the focus is primarily on customer care employees the same techniques can be used in other work settings as well. The words examinee, player and employee are used in the following paragraphs interchangeably.

\subsection{Gamified Assessment}

In the gamified assessment methods that are proposed here, the examinees would ideally use a mobile device like a smartphone or a tablet. They can instead use a desktop or their laptop to participate in these gamified assessments. The gamification software would typically be a software app developed on any of the popular platforms like android or iOS or Windows. Two or more examinees would login to the assessment app. Two examinees of almost same knowledge level would be paired together. Once a communication session is established between the two examinees, assessment would start.

The author has identified 10 different modes of conducting a gamified assessment. These modes are meant to add fun and offer variety to an employee's experience while he gets assessed.

\section{System driven}

In this mode the system would pose a question to both the examinees simultaneously. Examinees would get points based on who selects the correct answer first. The examinee who gave the correct answer first would get 2 points while the other examinee would get 1 point. A wrong answer will fetch 0 points. To avoid luck factor, negative marking for wrong answers may be introduced based on the domain of assessment.

This method can be further divided into two categories:

a. Fixed time span. The system would wait for a set amount of time, say 10 seconds, before moving on to the next question, even if one of the examinees has answered it before that time duration. The time duration for different questions may be different depending on the difficulty level of the question.

b. Early bird. In this method, the system would wait for at least one of them to answer the question. Once an examinee provides a correct answer it moves on to the next question without giving a chance for the opponent to give the answer. However, if the first examinee provided a wrong answer, the system will wait for the other examinee to provide his answer before moving on to the next question. Waiting time is subject to a maximum time span by which if neither of them has answered correctly they will both get 0 points for that question.

\section{Examinee driven}

In this mode, the system will allow an examinee to select a question from the question pool and let him pose it to the opponent. If the opponent could give a correct answer within a stipulated time, he gets one point, else he gets 0 . Now the opponent gets his chance to select a question from the question pool and pose it to his opponent. This way the players take turns and after a predetermined number of questions, their scores are compared to declare the winner.

This mode can further be subdivided into 2 modes:

a. Known answer. In this mode, a player is allowed to pose a question from the question pool only if he knows the correct answer to that question. He should answer it correctly before he is allowed to pass that question to his opponent.

b. Unknown answer: In this mode, the player is free to select any question from the question pool, whether he knows the correct answer to it or not.

\section{Third party driven}

In this mode, the examinees would wait for a third party, who could be another employee or a team leader, to pose a question to them. This mode can further be subdivided into two modes:

a. Simultaneous mode: In this mode, the question is posed to both the examinees simultaneously. The one who answers it correctly gets a point. The number of points assigned would be decided as in the system driven method described earlier.

b. Turn based mode: In this method, the third party would pose a question to one examinee. This would be followed by a question to the other employee. And the turns repeat.

\section{Round robin mode}

In this mode, examinees would be arranged in a round robin order. The turn starts with someone designated as first. The first examinee poses a question to the second examinee. After this turn, the second examinee poses a question to the third and so on till the last examinee poses a question to the first employee.

\section{Spectator participation mode}

a. Reward for spectators. To add more fun to the gamified assessment, other employees who are 'spectators' during a 'match' between 2 employees would also be allowed to participate in the ongoing match. If neither of the 2 players could answer a question posed by the system or a third party, the question passes to the spectators. Those who give the right answer within a stipulated time would get rewards. Here 


\section{International Journal of Science and Research (IJSR) \\ ISSN (Online): 2319-7064}

Index Copernicus Value (2013): 6.14 | Impact Factor (2015): 6.391

again, the early bird can be rewarded with more points than the ones who provided answers later.

b. Help from spectators. In this mode, spectators would be allowed to help an examinee if he could not answer a question, by letting them provide an answer from their smart devices. If the number of correct answers received is more than the number of wrong answers then that examinee earns a point otherwise he gets 0 points for that question. Here, there would be a limit on the percentage of questions for which an examinee can seek help from spectators.

\section{Collaborative mode}

In this mode, the examinees would be given the task of solving a specific customer issue. The solution would typically involve more than one step. The examinees would collaborate among themselves from their own devices to identify the correct steps to solve the given problem. The assessments in this category would typically focus on standard operating procedures and diagnostic procedures. The number of examinees would be at least two and it can more than two.

\section{Multiplayer mode}

In this mode, multiple players would form two teams. The teams will compete with each other in the same way that 2 players would compete.

\section{Boxing mode}

To add more fun to the assessment session, players can be allowed to pose more than one question, in succession without having to wait for the opponent to provide an answer to a question that is already posed. At the end of the session the player who has given correct answers to more number of questions wins. In this mode, a player would not be able to flood the opponent with too many questions as he himself has to earn more points to be declared a winner.

\section{Inter-organizational assessment competitions}

Inter-organizational competitions are common in variety of sports. Sports events are conducted where participants from different organizations compete with each other. Assessments can also be inter-organizational. They need not be confined to one organization. However, the question bank will be slightly different in nature. Questions that are too specific to an organization and for which answer is generally not available outside the organization should be avoided. Questions can be about the technology in general, it can be about a model of a product or a brand or about government's regulatory procedures or about procedures and processes that are common across organizations. All the modes discussed above can be applied to this scenario as well.

\section{Participation from friends and families}

In this mode, friends and families can offer help to the employee who is one of the competitors, in the same way as the case of spectators within the organization, which was discussed earlier. This approach can add a sense of closeness and belongingness to the employees towards the organization.

\section{Discussion}

We have seen several methods of assessing customer care employees using gamification. The primary aims of these methods of assessment are:

1. To make work fun and enjoyable for them and

2. To take the pressure out of them

Any or all of these methods can be used by an organization. To conduct such assessment sessions, the organizational team should have a complete list of questions that they want to include in their question. They should have an app that can work in the required mode. The employees generally possess smartphones and so this will not be a show stopper. Additionally, in the case of modes where spectators are allowed to participate, an equipment to project the question and the answers on a screen of some reasonable dimensions would be required.

\section{Future Scope}

The paper proposes several techniques of gamifying employee assessments. To make this a reality, the author intends to implement gamified assessment platforms in real life customer care scenarios. This would involve developing software apps on smartphone platforms like Android and iOS, implementing the modes of assessment proposed in the paper. It would also require a comprehensive question bank for employee assessment and a process for regular updates to the question bank to reflect the changes that happen to the business scenario.

\section{Conclusion}

We have shown how on-the-job assessments that are generally dreaded by employees can be made more of a fun and enjoyable activity in organizations. Ten different methods have been proposed and an organization can adopt any of the methods. The methods are particularly suitable for customer care employees who undergo tremendous pressure in their daily work routine.

\section{References}

[1] Weiss, D. J., \& Kingsbury, G. G. (1984). Application of computerized adaptive testing to educational problems. Journal of Educational Measurement, 21, 361-375.

[2] Huotari, K., \& Hamari, J. (2012). "Defining Gamification - A Service Marketing Perspective" (PDF). Proceedings of the 16th International Academic MindTrek Conference 2012, Tampere, Finland, October 3-5.

[3] Sicart, Miguel. "Defining Game Mechanics", http://gamestudies.org/0802/articles/sicart Retrieved on 16 October 2014.

[4] Brian Burke (2014) Gamify: How Gamification Motivates People to Do Extraordinary Things, Bibliomotion, Inc. Massachusetts. 


\section{Author Profile}

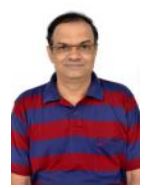

A.V. Murali received his B.Tech and M.Tech degrees in Metallurgical Engineering from IIT Madras. He has got an extensive work experience of over 25 years in diverse software application domains. His areas of work are primarily centered on applied research and systems implementation. His areas of research interest include software engineering, data mining and text mining, mathematical problem solving, gamification and game based learning.

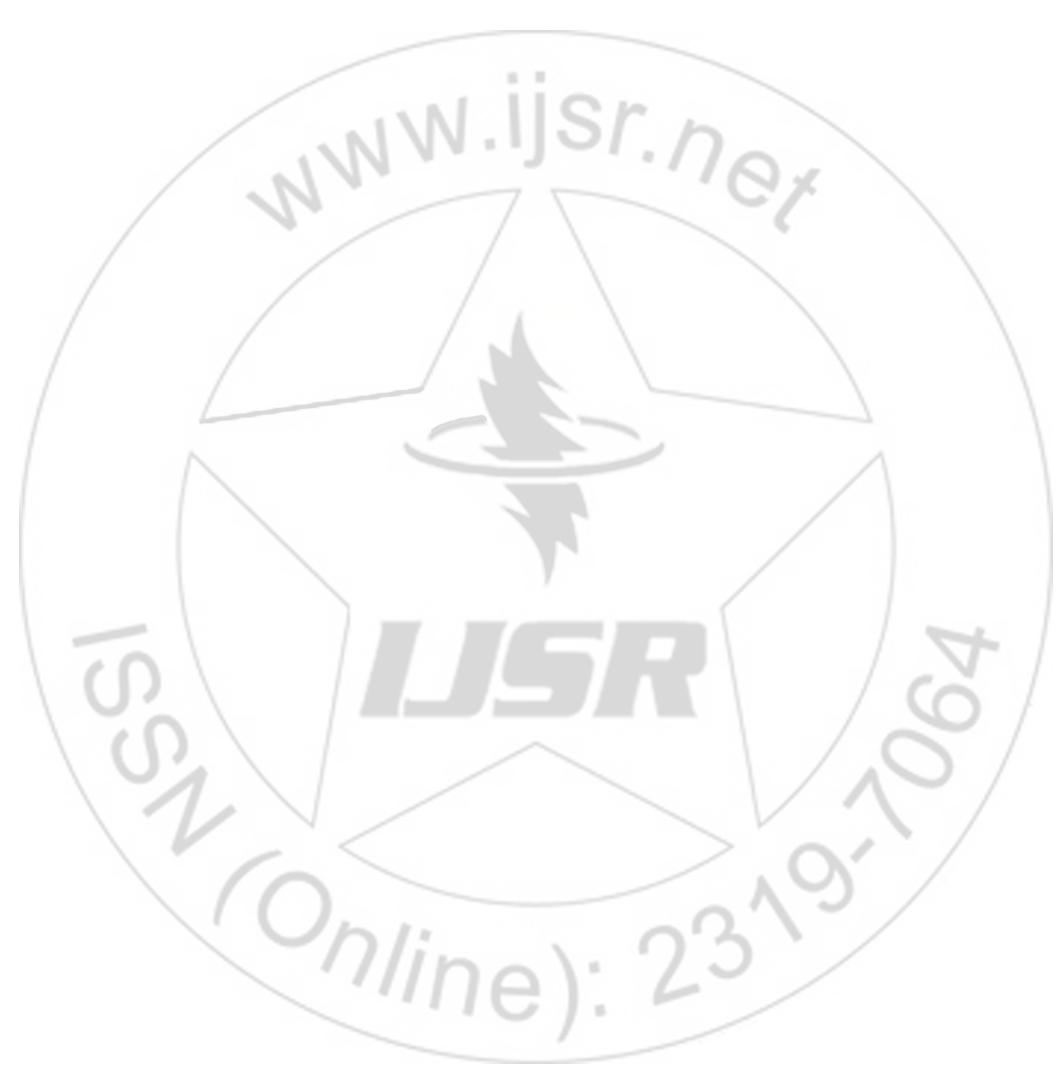

